\title{
Qualitative and Quantitative Features of Orbits of Massive Particles and Photons Moving in Wyman Geometry
}

\author{
G. Oliveira-Neto* \\ Departamento de Matemática e Computação, \\ Faculdade de Tecnologia, \\ Universidade do Estado do Rio de Janeiro, \\ Rodovia Presidente Dutra Km 298, Pólo Industrial, \\ CEP 27537-000, Resende, RJ, Brazil \\ G. F. Sousa ${ }^{\dagger}$ \\ Departamento de Física, Instituto de Ciências Exatas, \\ Universidade Federal de Juiz de Fora, \\ CEP 36036-330, Juiz de Fora, MG, Brazil \\ (Received on 20 July, 2008)
}

\begin{abstract}
The Wyman's solution depends on two parameters, the mass $M$ and the scalar charge $\sigma$. If one fixes $M$ to a positive value, say $M_{0}$, and let $\sigma^{2}$ take values along the real line it describes three different types of spacetimes. For $\sigma^{2}>0$ the spacetimes are naked singularities, for $\sigma^{2}=0$ one has the Schwarzschild black hole of mass $M_{0}$ and finally for $-M_{0}^{2} \leq \sigma^{2}<0$ one has wormhole spacetimes. In the present work, we shall study qualitative and quantitative features of orbits of massive particles and photons moving in the naked singularity and wormhole spacetimes of the Wyman solution. These orbits are the timelike geodesics for massive particles and null geodesics for photons. Combining the four geodesic equations with an additional equation derived from the line element, we obtain an effective potential for the massive particles and a different effective potential for the photons. We investigate all possible types of orbits, for massive particles and photons, by studying the appropriate effective potential. We notice that for certain naked singularities, there is an infinity potential wall that prevents both massive particles and photons ever to reach the naked singularity. We notice, also, that for certain wormholes, the potential is finite everywhere, which allows massive particles and photons moving from one wormhole asymptotically flat region to the other. We also compute the radial timelike and null geodesics for massive particles and photons, respectively, moving in the naked singularities and wormholes spacetimes.

Keywords: Wyman Geometry; Geodesics; Effective Potential; Naked Singularities and Wormholes
\end{abstract}

\section{INTRODUCTION}

The weak equivalence principle of general relativity tell us that massive particles move along timelike geodesics and photons move along null geodesics [1]. Despite their fundamental importance as one of the principles of general relativity, the geodesics also help us learning more about different properties of a given spacetime. A textbook example comes from the study of geodesics in Schwarzschild geometry [1]. Without actually computing the geodesics, just observing the effective potential diagram, one can see that both massive particles and photons can never leave the event horizon once they enter that surface. This is the case because the effective potential for both massive particles and photons diverges to negative infinity as one approaches the singularity located at the origin of the spherical coordinates. Therefore, once the massive particles and photons enter the event horizon they are accelerated toward the singularity without any chance to turn back. Many authors, over the years, have computed the effective potential diagram and the geodesics of different spacetimes in order to learn more about their properties. In particular, we may mention some important works dealing with different

\footnotetext{
*email:gilneto@fat.uerj.br

†email:gilberto_freitas@yahoo.com.br
}

black hole spacetimes [2]. Two other important gravitational configurations besides black holes that may form due to the gravitational collapse are naked singularities and wormholes. Some authors have already investigated some of their properties by computing effective potential diagrams and geodesics for those spacetimes [3-5]. A well-known spacetime geometry which may describe naked singularities as well as wormholes is the Wyman one [6]. Since, to the best of our knowledge, nobody has ever computed effective potential diagrams and geodesics for the Wyman spacetime, we decided to do that in the present work.

The Wyman geometry describes the four dimensional space-time generated by a minimally coupled, spherically symmetric, static, massless scalar field and has been studied by many authors [6-11]. From a particular case of the general Wyman's solution, M. D. Roberts showed how to construct a time dependent solution [8]. The Roberts' solution has an important physical interest because it may represent the gravitational collapse of a scalar field. Later, P. Brady and independently Y. Oshiro et al. [12], [13] showed that the Roberts' solution could be derived from the appropriated, time-dependent, Einstein-scalar equations by using a continuous self-similarity. They also showed that the Roberts' solution exhibits a critical behavior qualitatively identical to the one found numerically by M. W. Choptuik [14], studying the same system of equations. In fact, the above results confirmed early studies of D. Christodoulou who pioneered analytical 
studies of that model [15].

The Wyman's solution is not usually thought to be of great importance for the issue of gravitational collapse because it is static and the naked singularities derived from it are unstable against spherically symmetric linear perturbations of the system $[9,10]$. On the other hand, as we saw above, from a particular case of the Wyman's solution one may derive the Roberts' one which is of great importance for the issue of gravitational collapse. Also, it was shown that there are nakedly singular solutions to the static, massive scalar field equations which are stable against spherically symmetric linear perturbations [10]. Therefore, we think it is of great importance to gather as much information as we can about the Wyman's solution for they may be helpful for a better understand of the scalar field collapse.

The Wyman's solution depends on two parameters, the mass $M$ and the scalar charge $\sigma$. If one fixes $M$ to a positive value, say $M_{0}$, and let $\sigma^{2}$ take values along the real line it describes three different types of spacetimes. For $\sigma^{2}>0$ the spacetimes are naked singularities, for $\sigma^{2}=0$ one has the Schwarzschild black hole of mass $M_{0}$ and finally for $-M_{0}^{2} \leq \sigma^{2}<0$ one has wormhole spacetimes. Therefore, we have an interesting situation where we can study different properties of naked singularities and wormholes together through the effective potential diagrams and geodesics of massive particles and photons in the Wyman's solution.

In the next Section, we introduce the Wyman solution and identify the values of the parameters $M$ and $\eta$ that describes naked singularities, wormholes and the Schwarzschild black hole. In Section 3, we combine the geodesic equations to obtain the effective potential equation for the case of massive particles. We study the effective potential and qualitatively describe the types of orbits for massive particles moving in the naked singularities and wormholes spacetimes. In this section, we also compute the radial timelike geodesics for particles moving in the naked singularities and wormholes spacetimes. In Section 4, we combine the geodesic equations to obtain the effective potential equation for the case of photons. We study the effective potential and qualitatively describe the types of orbits for photons moving in the naked singularities and wormholes spacetimes. In this section, we also compute the radial timelike geodesics for photons moving in the naked singularities and wormholes spacetimes. Finally, in Section 5 we summarize the main points and results of our paper.

\section{NAKED SINGULARITIES, WORMHOLES AND THE} SCHWARZSCHILD BLACK HOLE.

The Wyman line element and the scalar field expression are given in coordinates $(t, r, \theta, \phi)$ by equation (9) of [8],

$$
\begin{gathered}
d s^{2}=-\left(1-\frac{2 \eta}{r}\right)^{\frac{M}{\eta}} d t^{2}+\left(1-\frac{2 \eta}{r}\right)^{-\frac{M}{\eta}} d r^{2}+ \\
+\left(1-\frac{2 \eta}{r}\right)^{1-\frac{M}{\eta}} r^{2} d \Omega^{2}
\end{gathered}
$$

where $r$ varies in the range $2 \eta<r<\infty$ and $d \Omega^{2}$ is the line element on the unit sphere. The scalar field is,

$$
\varphi=\frac{\sigma}{2 \eta} \ln \left(1-\frac{2 \eta}{r}\right)
$$

where $\sigma$ is the scalar charge given by $\sigma^{2}=\eta^{2}-M^{2}$ and $\sigma^{2} \geq$ $-M^{2}$. We are working in the unit system where $G=c=1$.

In the line element (1), the function $R(r)$,

$$
R(r)=r\left(1-\frac{2 \eta}{r}\right)^{\frac{1}{2}\left(1-\frac{M}{\eta}\right)}
$$

is the physical radius which gives the circumference and area of the two-spheres present in the Wyman geometry. Instead of using the parameters $M$ and $\sigma$ to identify the different spacetimes described by (1), we may also use the parameters $M$ and $\eta$, since they are all related by the equation just below (2). Each different spacetime will have a different behavior of $R(r) . R(r)$ has a single extremal value which is a minimum located at $r=M+\eta$. Since, the line element (1) is valid for $r>2 \eta$, only when $M>\eta$ there will be a minimum inside the domain of $r$. Based on that result we have three different cases for a positive $M$. As a matter of simplicity we shall use, also, the positive parameter $\lambda \equiv M / \eta$.

Case $M<\eta$ or $0<\lambda<1$.

In this case we have the following important values of $R(r)$ (3),

$$
\lim _{r \rightarrow 2 \eta_{+}} R(r)=0, \quad \lim _{r \rightarrow \infty} R(r)=\infty .
$$

Here, the solution represents spacetimes with a physical naked timelike singularity located at $R=0$ [7]. It is easy to see that this singularity is physical because, the Ricci scalar $\mathbf{R}$ computed from the line element (1),

$$
\mathbf{R}=\frac{2\left(M^{2}-\eta^{2}\right)}{r^{4}}\left(1-\frac{2 \eta}{r}\right)^{\frac{M}{\eta}-2}
$$

diverges there. It is also easy to see that this singularity is naked with the aid of the quantity $Q=g^{\alpha \beta} \frac{\partial R}{\partial x^{\alpha}} \frac{\partial R}{\partial x^{\beta}}$. The roots of $Q$ determine the presence of event horizons in spherical symmetric spacetimes [12]. For the Wyman solution $Q$ is given by,

$$
Q=\left(1-\frac{2 \eta}{r}\right)^{-1}\left(1-\frac{M+\eta}{r}\right)^{2}
$$

The only root of $Q$ is located at $r=M+\eta$ which confirms that the singularity located at $R=0$ is naked for all spacetimes in the present case.

This singularity is sometimes called a 'central singularity' and is similar to that appearing in the 'extreme' ReissnerNordstrøm black hole and in the negative mass Schwarzschild spacetime. From equation (2), the scalar field vanishes asymptotically as $R \rightarrow \infty$ and diverges at the singularity. 
Case $M=\eta$ or $\lambda=1$.

In this case we have $R(r)=r$ and $\sigma=0$. This last condition implies that the scalar field (2) vanishes and one gets the Schwarzchild solution. Here, the minimum of $R(r)$, located at $R=2 M$, is outside the domain of $r$ and the line element (1) describes only the spacetime exterior to the event horizon.

Case $M>\eta$ or $1<\lambda<\infty$. (3),

In this case we have the following important values of $R(r)$

$$
\lim _{r \rightarrow 2 \eta_{+}} R(r)=\infty, \quad R_{\min }=(M+\eta)\left(\frac{M-\eta}{M+\eta}\right)^{\frac{1}{2}\left(1-\frac{M}{\eta}\right)}, \quad \lim _{r \rightarrow \infty} R(r)=\infty .
$$

Due to the fact that $\sigma^{2} \geq-M^{2}$ as stated just before (2), we have that in the present case $\eta \geq 0$. The case $\eta=0$ is well know in the literature as the Yilmaz-Rosen space-time [16]. In this case $M>\eta$, the physical radius $R$ is never zero. If one starts with a large value of $R$, for large values of $r$, and starts diminishing $R$, reducing the values of $r$, one reachs the minimum value of $R\left(R_{\min }(7)\right)$ for $r=M+\eta$. Then, $R$ starts to increase again when we let $r$ goes to $2 \eta$ until it diverges when $r=2 \eta$. Therefore, we may interpret these spacetimes as wormholes connecting two asymptotically flat regions such that they have a minimum throat radius given by $R_{\min }(7)$ [11]. The spatial infinity $(R \rightarrow \infty)$ of each asymptotically flat region is obtained, respectively, by the limits: $r \rightarrow \infty$ and $r \rightarrow 2 \eta_{+}$. An important property of this space-time is that the scalar field (2) is imaginary. The imaginary scalar field also known as ghost Klein-Gordon field [17] is an example of the type of matter called exotic by some authors [18]. It violates most of the energy conditions and is repulsive. This property helps explaining the reason why the collapsing scalar field never reaches $R=0$.

As mentioned above, in the rest of the paper we shall restrict our attention to the spacetimes representing naked singularities and wormholes.

\section{TIMELIKE GEODESICS}

\subsection{Effective Potential}

We have four geodesic equations, one for each coordinate [1],

$$
\frac{d^{2} x^{\alpha}}{d \tau^{2}}+\Gamma_{\beta \gamma}^{\alpha} \frac{d x^{\beta}}{d \tau} \frac{d x^{\gamma}}{d \tau}=0
$$

where $\alpha=0,1,2,3$, and $x^{\alpha}$ represents, respectively, each of the coordinates $(t, r, \theta, \phi) . \tau$ is the proper time of the massive particle which trajectory is described by (8).

The geodesic equation for $\theta$ tell us that, like in the Schwarzschild case, the geodesics are independent of $\theta$, therefore we choose the equatorial plane to describe the particle motion $(\theta=\pi / 2)$. In the equatorial plane, the geodesic equation for $\phi$ can be integrated to give,

$$
r^{2}\left(1-\frac{2 \eta}{r}\right)^{1-\frac{M}{\eta}} \dot{\phi}=R^{2} \dot{\phi}=L
$$

where the dot means derivative with respect to $\tau$ and $L$ is the integration constant that may be interpreted as the particle angular momentum per unit rest mass. This result means that $\phi$ is cyclic and its conjugated momentum $\left(p_{\phi}=\dot{\phi}\right)$ is a conserved quantity. Also, $\dot{\phi}$ may be written as a function of $r$. Likewise, in the equatorial plane, the geodesic equation for $t$ can be integrated to give,

$$
\left(1-\frac{2 \eta}{r}\right)^{\frac{M}{\eta}} \dot{t}=E
$$

where $E$ is the integration constant that may be interpreted as the particle energy per unit rest mass. This result means that $t$ is cyclic and its conjugated momentum $\left(p_{t}=\dot{t}\right)$ is a conserved quantity. Also, $\dot{t}$ may be written as a function of $r$. Instead of using the fourth geodesic equation for $r$, we use the equation derived directly from the line element (1), $d s^{2} / d \tau^{2}=-1$ [19]. There, we introduce the expressions of $\dot{\phi}$ (9) and $\dot{t}$ (10), in order to obtain the following equation which depends only on $r$,

$$
\left(\frac{d r}{d \tau}\right)^{2}+V^{2}(r)=E^{2}
$$

Where

$$
V^{2}(r)=\left(1-\frac{2 \eta}{r}\right)^{\frac{M}{\eta}}\left[1+\frac{L^{2}}{r^{2}}\left(1-\frac{2 \eta}{r}\right)^{\frac{M}{\eta}-1}\right]
$$

and $V(r)$ is the effective potential for the motion of massive particles in the Wyman geometry. The geodesic equation for $r$ plays the role of a control equation, where we substitute the solutions to the other four equations, in order to verify their correctness.

In order to have a qualitative idea on the orbits of massive particles moving under the action of $V^{2}(r)$ it is important to learn more about it. We may do it by, initially, computing the extremal values of $V^{2}(r)$. First, we calculate the 
first derivative of $V^{2}(r)(12)$ and find the roots of the resulting equation. That equation may be simplified by the introduction of the auxiliary quantities: $x=(1-2 \eta / r), 0<x<1$; $A=\eta^{2} / L^{2}, 0<A<\infty ; B=(\lambda-1 / 2) /(\lambda+1 / 2),-1<B<1$; $C=2 \lambda /(\lambda+1 / 2), 0<C<2$. Where $\lambda$ was defined before and the domains of $A, B$ and $C$ where determined by the fact that $\lambda$ is positive. The equation $d V^{2}(r) / d r=0$, in terms of $x$, $\lambda, A, B$ and $C$ is given by,

$$
F(x)\left(A C x^{1-\lambda}+B-C x+x^{2}\right)=0,
$$

where $F(x)=\left[\lambda x^{\lambda-1}(1-x)\right]^{2} / 2 A C M$. Since $F(x) \neq 0$, for $0<x<1$, the term between parenthesis will give the roots of eq. (13). Unfortunately, there are not algebraic expressions for all these roots. The use of numerical techniques, in the present case, would give the roots of eq. (13) only for precise numerical values of all the parameters present in that equation. One would have to study each individual case, for each case is described by a different set of values of the parameters present in eq. (13). It is a possible way to solve eq. (13) but it would take a lot of computational time and effort. On the other hand, there is a mathematical treatment that allows the derivation of the algebraic expression of one root of eq. (13), in terms of $\lambda$. It, also, allows the identification of the exact number and nature (if it is a maximum, a minimum or a point of inflection of $V^{2}(r)$ ), besides the region in the $\lambda$ domain, of all the other roots of eq. (13). Furthermore, that mathematical treatment reduces greatly the computational time and effort, mentioned above. It starts with the definition of the following two auxiliary functions,

$$
p(x)=-x^{2}+C x-B, \quad h(x)=A C x^{1-\lambda} .
$$

Now, the values of $x$ where the two curves $p(x)$ and $h(x)$ meet will be the roots of (13). $p(x)$ is a set of parabolas which vertices are all located above the $\mathrm{x}$-axis and with two roots. The larger one is located at $x=1$ and the smaller one is located at $x=(\lambda-1 / 2) /(\lambda+1 / 2)$. For $\lambda>1 / 2$, the smaller root is positive; for $\lambda=1 / 2$, it is zero and for $\lambda<1 / 2$, it is negative. The precise nature of $h(x)$ will depend on the value of $\lambda$, present in the exponent of $x$, but whatever $\lambda$ one chooses, all values of $h(x)$ will be located above the $\mathrm{x}$-axis. For $\lambda>1, h(x)$ diverges to $+\infty$ when $x \rightarrow 0$ and goes to zero when $x \rightarrow+\infty$. For $\lambda<1, h(x)$ goes to zero when $x \rightarrow 0$ and diverges to $+\infty$ when $x \rightarrow+\infty$.

An important root of (13) is defined by the value of $x$, say $x_{0}$, where the two curves $p(x)$ and $h(x)$ (14) just touch each other. $x_{0}$ is a point of inflection of $V^{2}(r)(12)$, because there the second derivative of $V^{2}(r)$ is also zero. Associated to $x_{0}$ there is an angular momentum, say $L_{0}$, which originates an unstable particle orbit. Due to the fact that $L$ is present, only, in the denominator of $A$ in the expression of $h(x)$ (14), if one increases $L, h(x)$ will assume smaller values for the same values of $x . p(x)$ will not be altered because $L$ is not present in its expression. Therefore, $L_{0}$ is the value of $L$ for which $h(x)$ just touches $p(x)$, if one takes values of $L$ greater than $L_{0}, h(x)$ will start intercepting $p(x)$ in two or more points. They will be extremal values of $V^{2}(r)$ : maximum, minimum or point of inflection. It is possible to compute the value of $L_{0}$ in terms of $x_{0}$ and the parameters $\lambda$ and $\eta$. In order to do that, we consider, initially, the fact that the first derivatives of $h(x)$ and $p(x)(14)$, in $x=x_{0}$, are equal and express $A C x_{0}^{-\lambda}$ in terms of other quantities. Then, we use the fact that $x_{0}$ is a root of (13) and rewrite that equation for $x=x_{0}$ and substitute there the value of $A C x_{0}^{-\lambda}$ just obtained. It gives rise to the following second degree polynomial equation in $x_{0}$,

$$
x_{0}^{2}-\frac{C \lambda}{\lambda+1} x_{0}+\frac{\lambda-1}{\lambda+1} B=0,
$$

where $\lambda \neq 1$. It has the following roots,

$$
x_{0}^{+}=\frac{2 \lambda^{2}+\sqrt{5 \lambda^{2}-1}}{2(\lambda+1 / 2)(\lambda+1)}, \quad x_{0}^{-}=\frac{2 \lambda^{2}-\sqrt{5 \lambda^{2}-1}}{2(\lambda+1 / 2)(\lambda+1)},
$$

where $x_{0}^{+} \geq x_{0}^{-}$. Due to the fact that we have two distinct values of $x_{0}, x_{0}^{+}$and $x_{0}^{-}$given by (16), we shall have, also, two distinct values of $L_{0}$, say $L_{0+}$ and $L_{0-}$. In order to obtain them, we introduce $x_{0}^{+}$and $x_{0}^{-}$, separately, in the equation that equates the first derivatives of $h(x)$ and $p(x)$ and express $L_{0}^{2}$ in term of other quantities. It gives,

$$
\begin{aligned}
& L_{0+}^{2}=\frac{\eta^{2} \lambda(1-\lambda)}{\left(x_{0}^{+}\right)^{\lambda}\left[\lambda-x_{0}^{+}(\lambda+1 / 2)\right]}, \\
& L_{0-}^{2}=\frac{\eta^{2} \lambda(1-\lambda)}{\left(x_{0}^{-}\right)^{\lambda}\left[\lambda-x_{0}^{-}(\lambda+1 / 2)\right]},
\end{aligned}
$$

where $L_{0-}^{2} \geq L_{0+}^{2}$.

Since $x_{0}^{+}$and $x_{0}^{-}$vary in the range $[0,1]$ and $L_{0+}^{2}$ and $L_{0-}^{2}$ must be positive, we will have to impose some restrictions on the domain of $\lambda$. These restrictions lead to the following distinct domains of $\lambda$, depending on which root one is using,

$$
\begin{gathered}
x_{0}^{-}: \quad \frac{1}{\sqrt{5}} \leq \lambda<\frac{1}{2}, \\
x_{0}^{+}: \quad \lambda \geq \frac{1}{\sqrt{5}} .
\end{gathered}
$$

Therefore, this result tell us that $V^{2}(r)$ (12) behaves differently, depending on the value of $\lambda$. We have the following three different regions:

(i) $\lambda \geq 1 / 2$.

There will be just one point of inflection located at $x_{0}^{+}$for $L_{0+}^{2}$. If one chooses values of $L^{2}>L_{0+}^{2}$, one will find other extremal values of $V^{2}(r)(12)$, which will be a maximum or a minimum.

(ii) $1 / \sqrt{5} \leq \lambda<1 / 2$

There may be two different points of inflection. The first is located at $x_{0}^{+}$for $L^{2}=L_{0+}^{2}$. If one chooses values of $L^{2}>$ 
$L_{0+}^{2}$, one will find other extremal values of $V^{2}(r)(12)$, which will be a maximum or a minimum. When one reaches $L^{2}=$ $L_{0-}^{2}$, one finds the other possible point of inflection located at $x_{0}^{-}$. If one chooses values of $L^{2}>L_{0-}^{2}$, one will find just one extremal value of $V^{2}(r)$ (12), which will be a minimum. Even for the case $L^{2}<L_{0+}^{2}$, there will be a minimum. In fact, this minimum will always be present for any value of $L$. Its presence can be understood because, here, $p(x)$ has a negative root and $h(x)$ is crescent and starts from $x=0$. Therefore, these two curves will always intercept each other.

(iii) $\lambda<1 / \sqrt{5}$.

There will be no point of inflection but there will be always a minimum. The presence of this minimum can be understood in the same way as the one in the previous case.

As we saw in the previous section, Sec. 2, naked singularities and wormholes are characterized by certain subdomains of $\lambda$. Therefore, based on the above result, we may describe the effective potentials for naked singularities and wormholes.

\subsection{Effective Potential for Naked Singularities}

As we saw, in Sec. 2, the naked singularities are obtained for $0<\lambda<1$. We may still divide the naked singularities in two classes, due to the behavior of $V^{2}(r)$ (12) when $r \rightarrow 2 \eta_{+}$. Since the present naked singularity is a physical singularity, we expect that $V^{2}(r)$ diverges there.

For $0<\lambda<1 / 2, \lim _{r \rightarrow 2 \eta_{+}} V^{2}(r)=\infty$. Due to the fact that this limit is consistent with an asymptotically flat naked singularity located at $r=2 \eta$, we call this class of ordinary naked singularities.

On the other hand, for $1 / 2 \leq \lambda<1, \lim _{r \rightarrow 2 \eta_{+}} V^{2}(r)=0$. Due to fact that this result is not consistent with a naked singularity located at $r \rightarrow 2 \eta$, we call this class of anomalous naked singularities. Then, in what follows we shall restrict our attention to the class of ordinary naked singularities with $0<\lambda<1 / 2$. It is important to mention that observing the scalar field expression (2), which in this case is real, one can see that it diverges to $-\infty$ as $r \rightarrow 2 \eta_{+}$.

Taking in account the results of Subsec. 3.1 (ii) and (iii) the effective potential $V^{2}(r)$ (12) may have several different shapes depending on the value of $L^{2}$. Here, the points of inflection will be located at $x_{0}^{-}$and $x_{0}^{+}(16)$ and the relevant angular momenta will be $L_{0-}^{2}$ and $L_{0+}^{2}(17)$.

- For $L^{2}<L_{0+}^{2}, V^{2}(r)(12)$ has one minimum. In terms of $x$ it is located in the range $\left(0, x_{0}^{-}\right)$or in terms of $r$ it is located in the range $\left(2 \eta, r_{0}^{-} \equiv 2 \eta /\left(1-x_{0}^{-}\right)\right)$. For $E^{2}<1$, the massive particles orbit around the naked singularity. If the massive particle is located exactly at the minimum the orbit is circular and stable. For $E^{2}>1$, the massive particles come in from infinity reach the infinity potential wall near the naked singularity and return to infinity without ever reach the naked singularity.

- For $L^{2}=L_{0+}^{2}, V^{2}(r)$ (12) has two extremal values, a minimum located in the range $\left(2 \eta, r_{0}^{-}\right)$and a point of inflection located at $r=r_{0}^{+} \equiv 2 \eta /\left(1-x_{0}^{+}\right)$. In this point the massive particles have unstable circular orbits. The other possible trajectories for the massive particles are exactly as in the previous case.

- For $L_{0+}^{2}<L^{2}<L_{0-}^{2}, V^{2}(r)(12)$ has three extremal values, a minimum located in the range $\left(2 \eta, r_{0}^{-}\right)$, a maximum located in the range $\left(r_{0}^{-}, r_{0}^{+}\right)$and another maximum located in the range $\left(r_{0}^{+}, \infty\right)$. If the massive particles are exactly in the maxima they have unstable circular orbits. The other possible trajectories for the massive particles are exactly as in the first case.

- For $L^{2}=L_{0-}^{2}, V^{2}(r)$ (12) has two extremal values, a minimum located in the range $\left(r_{0}^{+}, \infty\right)$ and a point of inflection located at $r=r_{0}^{-}$. In this point the massive particles have unstable circular orbits. The other possible trajectories for the massive particles are exactly as in the first case.

- For $L^{2}>L_{0-}^{2}, V^{2}(r)(12)$ has one minimum located in the range $\left(r_{0}^{+}, \infty\right)$. The possible trajectories for the massive particles are exactly as in the first case.

It is important to mention that, for sufficiently large values of $E$, any massive particles will come in from infinity and it will get reflected by the infinity potential wall near the naked singularity. Then, it will return to infinity without ever reach the naked singularity. This result is similar to the one found in [4] for timelike geodesics of the naked singularity present in the Reissner-Nordström spacetime. Let us consider, as an example, the case where $M=10$ and $\lambda=0.45$. Therefore, we have $L_{0+}^{2}=954.9308516$ and $x_{0}^{+}=0.1875874406$ which in terms of $r$ is given by $r_{0}^{+}=54.70674220$. We also have, $L_{0-}^{2}=$ 960.0309665 and $x_{0}^{-}=0.1064234487$ which in terms of $r$ is given by $r_{0}^{-}=49.73770224$. In Fig. 1 , we plot $V^{2}(r)$ (12) for each of the five cases discussed above. They have the five different values of $L^{2}: 951,954.9308516,957,960.0309665$ and 962 . The naked singularity is located at $r=44.44444444$.

\subsection{Effective Potential for Wormholes}

As we saw, in Sec. 2, the wormholes are obtained for $1<\lambda<\infty$. In the spatial infinity of each asymptotically flat region the effective potential $V^{2}(r)$ (12) assumes the following values,

$$
\lim _{r \rightarrow 2 \eta_{+}} V^{2}(r)=0, \quad \lim _{r \rightarrow \infty} V^{2}(r)=1 .
$$

Although the above limits give consistent values for the effective potential at the two spatial infinities, the first limit is not consistent with the value of the Ricci scalar evaluated at $r=2 \eta$, for wormholes with $1<\lambda \leq 2$. For this class of wormholes the Ricci scalar $\mathbf{R}$ (5), diverges to $\infty$ when we take the limit $r \rightarrow 2 \eta_{+}$or gives a positive constant when $\lambda=2$. These values are not consistent with the first limit of $V^{2}(r)$ in (20) and the idea of an asymptotically flat spatial region. Therefore 


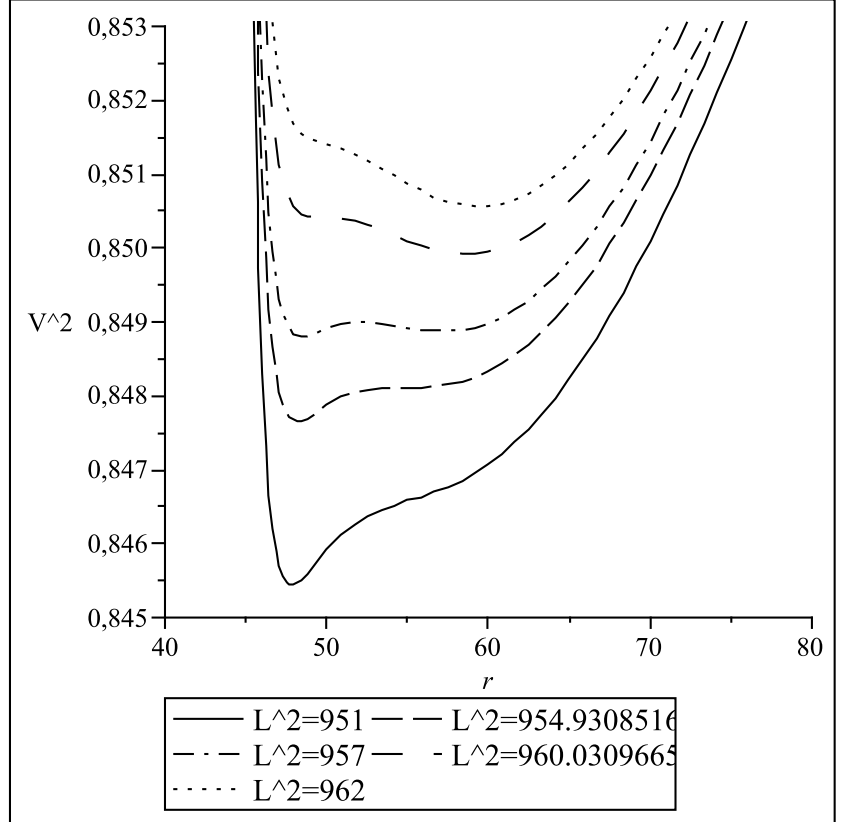

FIG. 1: Five different effective potential diagrams $V^{2}(r)$ (12), for massive particles moving in a naked singularity with $M=10$ and $\lambda=$ 0.45 . They have the five different values of $L^{2}: 951,954.9308516$, $957,960.0309665$ and 962 . The naked singularity is located at $r=$ 44.44444444.

we call this class of wormholes of anomalous wormholes. For the rest of the wormholes where $2<\lambda<\infty$, the limit of $\mathbf{R}$ (5) when $r \rightarrow 2 \eta_{+}$is zero. This value is consistent with the first limit of $V^{2}(r)$ in (20) and the idea of an asymptotically flat spatial region. Then, in what follows we shall restrict our attention to that class of ordinary wormholes with $2<\lambda<\infty$.

Taking in account the results of Subsec. 3.1 (i) the effective potential $V^{2}(r)$ (12) may have three different shapes depending on the value of $L^{2}$. Here, the point of inflection will be located at $x_{0}^{+}(16)$ and the relevant angular momentum will be $L_{0+}^{2}$ (17).

- For $L^{2}<L_{0+}^{2}, V^{2}(r)(12)$ has no extremal values. In this case, there is no stable orbits. For sufficiently high energies the massive particles may travel from one asymptotically flat region to the other. In fact, this type of orbit is also present in the next two cases.

- For $L^{2}=L_{0+}^{2}, V^{2}(r)$ (12) has one point of inflection, located at $x_{0}^{+}(16)$ or in terms of $r, r_{0}^{+} \equiv 2 \eta /\left(1-x_{0}^{+}\right)$. In this point the massive particles have unstable circular orbits.

- For $L^{2}>L_{0+}^{2}, V^{2}(r)$ (12) has two extremal values, a maximum located at $2 \eta<r<r_{0}^{+}$and a minimum located at $r_{0}^{+}<r$. There are closed and open orbits depending on the values of the total energy and angular momentum of the massive particles.

Let us consider, as an example, the case where $M=1$ and $\lambda=\sqrt{1000}$. Therefore, we have $L_{0+}^{2}=12.41266$ and

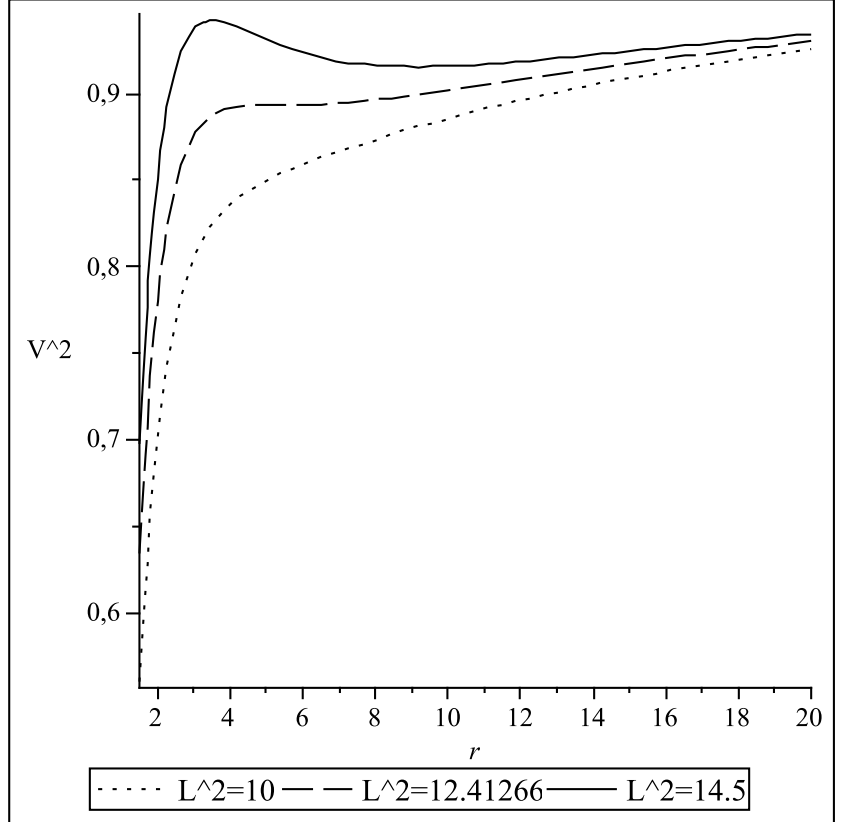

FIG. 2: Three different effective potential diagrams $V^{2}(r)$ (12), for massive particles moving in a wormhole with $M=1$ and $\lambda=\sqrt{1000}$. They have the three different values of $L^{2}: 10,12.41266$ and 14.4. The spatial infinities of each asymptotically flat region are located at $r=0.06325$ and $r \rightarrow \infty$.

$x_{0}^{+}=0.98799$ which in terms of $r$ is given by $r_{0}^{+}=5.26747$. In Fig. 2, we plot $V^{2}(r)$ (12) for each of the three cases discussed above. They have the three different values of $L^{2}: 10$, 12.41266 and 14.4. The spatial infinities of each asymptotically flat region are located at $r=0.06325$ and $r \rightarrow \infty$.

\subsection{Radial timelike geodesics}

Unfortunately, there is not an algebraic expression for the general timelike geodesics given by the solutions to Eqs. (9$11)$, even the numerical solutions are very complicated. On the other hand, one may restrict his attention to the case of radial timelike geodesics, where the massive particle moves only along the radial and time directions. It means that $\dot{\theta}=$ $\dot{\phi}=L=0$ and (11) reduces to,

$$
\left(\frac{d r}{d \tau}\right)^{2}=E^{2}-\left(1-\frac{2 \eta}{r}\right)^{\frac{M}{\eta}} .
$$

Although (21) is much simpler than (11), one still cannot obtain an algebraic expression of $\tau$ as a function of $r$, after its integration. Therefore, we shall perform a numerical study of the solutions to (21) and present our results.

a. Naked singularities In this case, we integrated (21) for many different values of $E, M$ and $\eta$. We chose values of $M$ and $\eta$ compatibles with naked singularities. We found that, for $E \geq 1$, the geodesics are all well behaved when $r \rightarrow 2 \eta$. In fact, $\tau$ goes to zero in that limit. The geodesics are such that 
when $r$ is large, $r$ tends to a linear function of the proper time $\tau$. For $E<1$, the geodesics are also well behaved when $r \rightarrow 2 \eta$ and $\tau$ also goes to zero in that limit. On the other hand, they do not extend to large values of $r$. It is clear from (21), that the massive particles are subjected to a potential of the form: $(1-$ $2 \eta / r)^{M / \eta}$. This potential, increases from zero at $r=2 \eta$ and tends to one when $r \rightarrow \infty$. Therefore, massive particles with a total energy $E<1$, get reflected by the potential. The value of $r$, where the particle gets reflected by the potential is obtained by solving the equation: $E^{2}=(1-2 \eta / r)^{M / \eta}$. Then, based on the above results, we may conclude that it always takes a finite proper time interval to travel from any finite value of $r$ to the singularity located at $r=2 \eta$. We notice, also, that for radial timelike geodesics there is not an infinity potential wall near the naked singularity and the massive particles can reach it.

b. Wormholes In this case, we integrated (21) for many different values of $E, M$ and $\eta$. We chose values of $M$ and $\eta$ compatibles with wormholes. We found that, for $E \geq 1$, the geodesics are all well behaved when $r \rightarrow 2 \eta$. In fact, $\tau$ goes to zero in that limit. The geodesics are such that when $r$ is large, $r$ tends to a linear function of the proper time $\tau$. For $E<1$, the geodesics are also well behaved when $r \rightarrow 2 \eta$ and $\tau$ also goes to zero in that limit. On the other hand, they do not extend to large values of $r$ because they get reflected by the potential, as in the naked singularity case. The value of $r$, where the particle gets reflected by the potential is obtained in the same way as in the naked singularity case. Here, as in the naked singularity case, for all cases studied it always takes a finite proper time interval to travel from any finite value of $r$ to the spatial infinity located at $r=2 \eta$.

\section{NULL GEODESICS}

\subsection{Effective Potential}

The null geodesics for the Wyman solution are derived almost in the same way the timelike geodesics were derived in the previous section. The only difference is that, here, the null line element contributes a different additional equation to the four geodesic equations. The new equation reads: $d s^{2} / d \chi^{2}=0$, where $\chi$ is the affine parameter used in the present case. Therefore, proceeding exactly as in the previous section we obtain the following effective potential equation,

$$
\left(\frac{d r}{d \chi}\right)^{2}+V^{2}(r)=\frac{1}{b^{2}},
$$

where

$$
V^{2}(r)=r^{-2}\left(1-\frac{2 \eta}{r}\right)^{\frac{2 M}{\eta}-1}
$$

and $b \equiv L / E$ is the photon impact parameter. $V^{2}(r)(23)$ is the effective potential for the motion of the photon in Wyman geometry.
$V^{2}(r)$ (23) has only one extremal value at $r=2 M+\eta$. Due to the domain of $r$ it can only exists if $2 M>\eta$ or $\lambda>1 / 2$ and when it exists it is a maximum. Therefore, we have three different cases: (i) $\lambda>1 / 2$, the effective potential has a maximum at $r=2 M+\eta$ and goes to zero at $r=2 \eta$;(ii) $\lambda=1 / 2$, the effective potential has no extremal values and diverges to $\infty$ as $r \rightarrow 0$; (iii) $\lambda<1 / 2$, the effective potential has no extremal values and diverges to $\infty$ as $r \rightarrow 2 \eta$.

\subsection{Effective Potential for Naked Singularities}

As it was mentioned in Subsec. 3.2, we are only concerned, here, with the class of ordinary naked singularities characterized $0<\lambda<1 / 2$. For this class, $V^{2}(r)$ (12) assumes the following values when $r \rightarrow 2 \eta_{+}$and $r \rightarrow+\infty$,

$$
\lim _{r \rightarrow 2 \eta_{+}} V^{2}(r)=\infty, \quad \lim _{r \rightarrow+\infty} V^{2}(r)=0
$$

$V^{2}(r)$ (23) has no extremal values. In this case, whatever the impact parameter $b$ the photons come in from infinity, get reflected by the infinity potential wall near the naked singularity and return to infinity without ever reach the naked singularity.

\subsection{Effective Potential for Wormholes}

As it was mentioned in Subsec. 3.3, we are only concerned, here, with the class of ordinary wormholes characterized by $2<\lambda<\infty$. In the spatial infinity of each asymptotically flat region $V^{2}(r)$ (23) assumes the following values,

$$
\lim _{r \rightarrow 2 \eta_{+}} V^{2}(r)=0, \quad \lim _{r \rightarrow \infty} V^{2}(r)=0 .
$$

$V^{2}(r)$ (23) has a maximum located at $r=2 M+\eta$. In this point the photons have unstable circular orbits. For sufficiently small impact parameter $b$ the photons may travel from one asymptotically flat region to the other. Otherwise, they come in from spatial infinity of an asymptotically flat region get reflected by the effective potential and return to spatial infinity in the same asymptotically flat region.

\subsection{Radial null geodesics}

The description of the null geodesics in terms of the affine parameter $\chi$ is trivial, when we consider radial geodesics $(\dot{\theta}=\dot{\phi}=L=0)$. That is the case because the effective potential equation for the radial motion of photons reduces to $(d r / d \chi)^{2}=E^{2}$. It means that the effective potential is zero and the photons move freely following straight lines: $r= \pm E \chi+q$, where $q$ is an integration constant and the + and - signs mean, respectively, outgoing and ingoing geodesics. This result is valid for photons moving in the naked singularity as well as in the wormhole geometries. 


\section{CONCLUSIONS}

In the present work, we studied qualitative and quantitative features of orbits of massive particles and photons moving in the naked singularity and wormhole spacetimes of the Wyman solution. We investigated all possible types of orbits, for massive particles and photons, by studying the appropriate effective potential. We noticed that for certain naked singularities, there is an infinity potential wall that prevents both massive particles and photons ever to reach the naked singularity. This result is similar to the one found in [4] for timelike geodesics of the naked singularity present in the Reissner-Nordström spacetime. We noticed, also, that for certain wormholes, the potential is finite everywhere, which allows massive particles and photons moving from one wormhole asymptotically flat region to the other. We also computed the radial timelike and null geodesics for massive particles and photons, respectively, moving in the naked singularities and wormholes spacetimes. It is important to mention that, the above description of the radial timelike and null geodesics in terms of $\tau$ and $\chi$ may also be done in terms of the time coordinate $t$, leading to few different results [20].

Acknowledgments. G. Oliveira-Neto (Researcher of $\mathrm{CNPq}$, Brazil) thanks CNPq and FAPERJ for partial financial support and G F Sousa thanks CAPES for financial support.
[1] For a detailed explanation see: C. W. Misner, K. S. Thorne and J. A. Wheeler, Gravitation, (Freeman, New York, 1973).

[2] M. J. Jaklitsch, C. Hellaby, and D. R. Matraversl, Gen. Rel. Grav. 21, 941 (1989); Z. Stuchlík and M. Calvani, Gen. Rel. Grav. 23, 507 (1991); Z. Stuchlík and S. Hledík, Phys. Rev. D 60, 044006 (1999); J. Podolský, Gen. Rel. Grav. 31, 1703 (1999); G. V. Kraniotis and S. B. Whitehouse, Class. Quant. Grav. 20, 4817 (2003); G. V. Kraniotis, Class. Quant. Grav. 21, 4743 (2004); N. Cruz, M. Olivares, and J. R. Villanueva, Class. Quant. Grav. 22, 1167 (2005).

[3] J. M. Cohen and R. Gautreau, Phys. Rev. D 19, 2273 (1979); Z. Stuchlík and S. Hledík, Class. Quant. Grav. 17, 4541 (2000); Z. Stuchlík and P. Slaný, Phys. Rev. D 69, 064001 (2004).

[4] A. Qadir and A. A. Siddiqui, Int. J. Mod. Phys. D 16, 25 (2007).

[5] J. C. Graves and D. R. Brill, Phys. Rev. 120, 1507 (1960); M. Azreg-Ainou and G. Clément, Gen. Rel. Grav. 22, 1119 (1990).

[6] M. Wyman, Phys. Rev. D 24, 839 (1981).

[7] I. Z. Fisher, Zh. Eksp. Teor. Fiz. 18, 636 (1948) and an English version available in gr-qc/9911008; O. Bergman and R. Leipnik, Phys. Rev. D 107, 1157 (1957); H. A. Buchdahl, ibid. 111, 1417 (1959); A. I. Janis, E. T. Newman, and J. Wincour, Phys. Rev. Lett. 20, 878 (1968); J. E. Chase, Commun. Math. Phys. 19, 276 (1970); A. G. Agnese and M. LaCamera, Lett. Nuovo Cimento 35, 365 (1982); Phys. Rev. D 31, 1280 (1985); D. D. Dionysion, Astro. Space Sci. 83, 493 (1982); J. Froyland, Phys. Rev. D. 25, 1470 (1982); M. D. Roberts, Gen. Rel. Grav. 17,
913 (1985); Class. Quant. Grav. 2, L69 (1985); Astro. Space Sci. 147, 321 (1988).

[8] M. D. Roberts, Gen. Rel. Grav. 21, 907 (1989).

[9] P. Jetzer and D. Scialom, Phys. Lett. A 169, 12 (1992).

[10] M. A. Clayton, L. Demopoulos, and J. Legare, Phys. Lett. A 248, 131 (1998).

[11] G. Oliveira-Neto and F. I. Takakura, J. Math. Phys. 46, 062503 (2005).

[12] P. R. Brady, Class. Quant. Grav. 11, 1255 (1994).

[13] Y. Oshiro, K. Nakamura, and A. Tomimatsu, Prog. Theor. Phys. 91, 1265 (1994).

[14] M. W. Choptuik, Phys. Rev. Lett. 70, 9 (1993).

[15] D. Christodoulou, Commun. Math. Phys. 105, 337 (1986); 106, 587 (1986); 109, 591 and 613 (1987); Commun. Pure Appl. Math. XLIV 339 (1991); XLVI, 1131 (1993); Ann. Math. 140, 607 (1994).

[16] H. Yilmaz, Phys. Rev. 111, 1417 (1958); N. Rosen, in Relativity, eds. Carmeli, Fickler and Witten (New York, N. Y., 1970), 229.

[17] S. A. Hayward, S. W. Kim, and H. Lee, Phys. Rev. D 65, 064003 (2002).

[18] M. S. Morris and K. S. Thorne, Am. J. Phys. 56, 395 (1988).

[19] R. A. D'Inverno, Introducing Einstein's Relativity, (Oxford University Press, Oxford, England, 1992).

[20] G. Oliveira-Neto and G. F. Sousa, gr-qc/08014531. 